\title{
Consensus PCR
}

National Cancer Institute

\section{Source}

National Cancer Institute. Consensus PCR. NCI Thesaurus. Code C127879.

Polymerase chain reaction that uses primers designed to amplify sequences from a group of genetically related targets, using specific or degenerate primer sets. 